\title{
Asymptotic Forms for the Whittaker \\ Functions with Both Parameters Large
}

\author{
NIGHOLAS D. KAZARINOFF ${ }^{1}$
}

Communicated by A. ERDÉLyI

1. Introduction. Certain solutions of the differential equation

$$
\frac{d^{2} W}{d x^{2}}+\left\{-\frac{1}{4}+\frac{k}{x}+\frac{\frac{1}{4}-m^{2}}{x^{2}}\right\} W=0
$$

are called Whittaker functions and are customarily designated $W_{k, m}(x)$ and $M_{k, m}(x)$. Their asymptotic behavior is different according as one, two, or three of the quantities $|x|,|k|$, and $|m|$ are large. Recent books by H. BuchHolz [2] and F. Tricomi [10] contain summaries of many investigations in this area. Other results are to be found in $[3,4,5,6]$. An examination of this literature reveals that least is known of the structure of the Whittaker functions if both $k$ and $m$ are unbounded. When $|x|$ is also large, such information as is available applies only to nearly real values of $x$ and $k$ and nearly real or pure imaginary values of $m$ and is subject to the further limitation that the ratios $x / k$ and $x / m$ be nearly fixed $[3,4,5]$.

In this paper the asymptotic behavior of the Whittaker functions with $x, k$, and $m$ real or complex is obtained for both $|k|$ and $|m|$ large and $x$ unrestricted under the hypothesis that $\left(m^{2}-k^{2}\right) / k$ be bounded, so that $\lim _{|k| \rightarrow \infty} m / k= \pm 1$. The results are stated in Theorems $1,2,4$, and 5 of the sequel. The analysis to be given is based upon the methods of asymptotic solution of ordinary differential equations containing a large parameter developed by R. E. LANGER. The author hopes to investigate the behavior of the Whittaker functions for other configurations of large $k$ and $m$ subsequently, using these same methods. In this paper particular reference is made to work of R. McKelver [9]. The saddlepoint and power series methods have been the principal tools in previous investigations $[3,4,5,10]$. It should be remarked that in the notation of the confluent

\footnotetext{
1 This research was supported by the United States Air Force through the Office of Scientific Research, Air Research and Development Command under contract No. AF 18(600)-1481.
} 
hypergeometric functions ${ }_{1} F_{1}(a ; c ; x)$ and ${ }_{1} F_{1}(1+a-c ; 2-c ; x)$ the configuration of $k$ and $m$ considered here is equivalent to that of $|c| \rightarrow \infty$ while $a$ is bounded. In view of the long history of Kummer's function ${ }_{1} F_{1}(a ; c ; x)$ it is rather surprising that so little is known of its behavior as $|c| \rightarrow \infty[1,10]$. It is for simplicity of presentation that the notation of WhITTAKER is used here in in preference to that of Kummer.

The form of WhitTakeR's equation (1.1) most suitable for the application of McKelvey's theory [9] is obtained by making the transformations

$$
\begin{aligned}
& z=\ln (x / 2 k)=\ln |x / 2 k|+i \arg (x / 2 k), \\
& u=e^{-\frac{1}{2} z} W .
\end{aligned}
$$

These reduce (1.1) to the form

$$
\frac{d^{2} u}{d z^{2}}-\left\{k^{2}\left(e^{2}-1\right)^{2}+k \sigma\right\} u=0
$$

with

$$
\sigma=\left(m^{2}-k^{2}\right) / k .
$$

The principal feature of this equation is the presence of a second order turning point at $z=0(x=2 k)$. Near this turning point it is necessary to use McKelveY's analysis, extended to a complex domain, in the study of solutions of (1.3). Unfortunately, for reasons most conveniently stated at the end of $\$ 6$, the extended analysis cannot be used when $R(z) \rightarrow+\infty$; and hence for large $R(z)$ the structure of solutions of (1.3) will be investigated by means of the classical theory of asymptotic solution near an ordinary point of ordinary differential equations containing a large parameter. For $R(z) \rightarrow-\infty$, i.e., small values of $|x / 2 k|$, the situation is simple [1,2] as the hypergeometric representations for $M_{k, \pm m}(x)$ are rapidly convergent asymptotic series in $m$. However, as $|x / 2 k| \rightarrow 1$, the hypergeometric series converge less rapidly and the need for other asymptotic expansions arises.

A broad outline of the paper is as follows. In $\S 2$ such restrictions on $x$ and $k$ as can be made without loss of generality are set forth and the properties of the assumed configurations of $x$ and $k$ with respect to certain mappings are discussed. In $\$ 3$ the asymptotic behavior of the Whittaker functions is determined for $|k|,|m|$, and $|x / 2 k|$ large and $\sigma$ bounded. In $\$ \$ 4-7$ McKelvey's theory is extended to a complex domain for equation (1.3). The extension gives the asymptotic behavior of two linearly independent solutions of (1.3) for $|x / 2 k|$ close to 1. Lastly, in $\S 8$ the connection formulas among these solutions and the solutions of (1.3) described in $\S 3$ are determined and the asymptotic behavior of the Whittaker functions near the turning point $x=2 k$ thereby discovered.

It is convenient to introduce at this point some notation which will be used repeatedly hereafter. The letters $M$ and $N$ are always used as generic symbols denoting positive constants. The letter $E$ is always used as a generic symbol for a bounded function of one or more variables. 
The configurations of real, numerically large $k$ and $m$, including the one considered here, for which the structure of the Whittaker functions has been determined for arbitrary $|x|$ are illustrated in the shaded regions of Fig. 1.

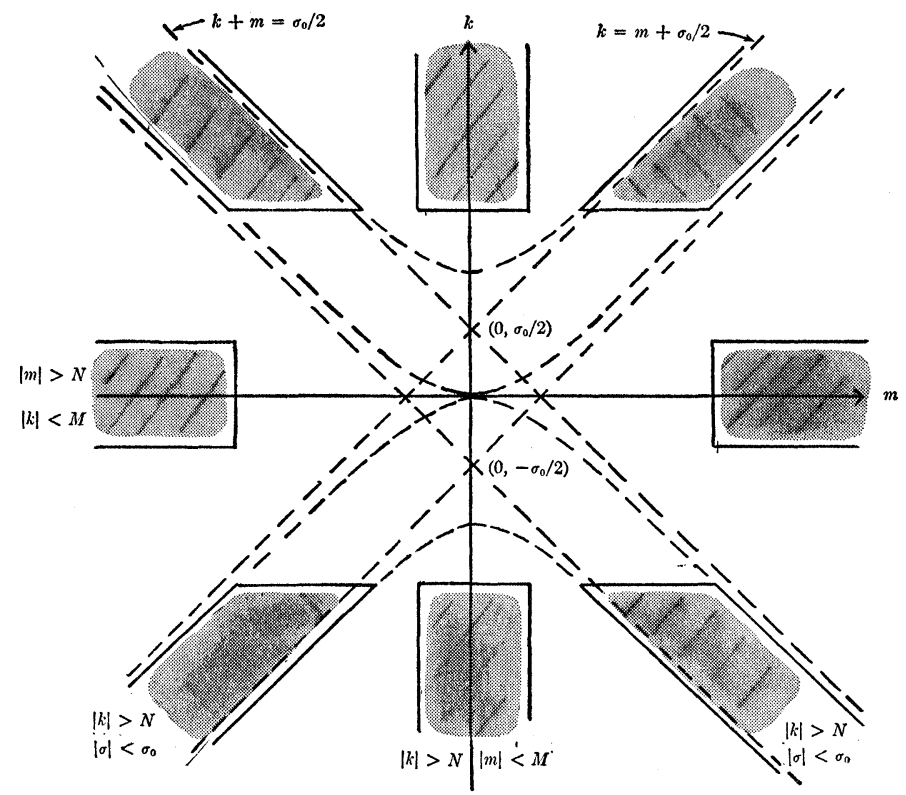

FIG. 1.

2. Preliminaries. Let $\alpha$ and $\beta$ be positive numbers such that $\alpha>\beta$. For the remainder of this paper it will be assumed that either

$$
e^{-\alpha} \leqq|x / 2 k| \leqq e^{\alpha}, \quad-\pi<\arg x \leqq \pi, \quad \text { and } \quad 0 \leqq \arg k \leqq \pi
$$

or

$$
|x / 2 k| \geqq e^{\beta}, \quad 0 \leqq \arg x \leqq \pi, \quad \text { and } \quad|\arg k| \leqq \pi .
$$

Asymptotic forms for the functions $W_{k, m}(x)$ and $W_{-k, m}\left(e^{-\pi i} x\right)$ will be determined subject to these restrictions. Once their behavior for the values of $x$ and $k$ allowed by (2.1) is known, the behavior of $W_{k, m}(x)$ for $-\pi<\arg x \leqq \pi$ and $|\arg k| \leqq \pi$ will also be at hand [2,\$2, formula (37a)]. Also, if their behavior is known for the values of $x$ and $k$ allowed by (2.2), the behavior of $W_{k, m}(x)$ for $|\arg x| \leqq \pi$ and $|\arg k| \leqq \pi$ will be at hand. This means that the asymptotic form of two linearly independent solutions of (1.1) will be available over a range of $2 \pi$ in both the arguments of $x$ and $k$. Thus there is no loss of generality in imposing the restrictions (2.1) and (2.2).

Apparently multiple-valued functions of the form $f^{a}$ often occur in the sequel. It is assumed, once for all, that $f^{a}=\exp [a(\ln |f|+i \arg f)]$. $\operatorname{Arg} f$ will always be single-valued. 
Under the change of variable in (1.2) the $x$-plane cut along arg $x= \pm \pi$ is mapped conformally onto the horizontal strip of the $z$-plane defined by

$$
-\pi-\arg k<\mathscr{g}(z) \leqq \pi-\arg k .
$$

In order to preserve the single-valuedness of nonintegral powers of $z$ and $(x-2 k)$ it is further assumed that

$$
-\pi<\arg z \leqq \pi
$$

so as to cut the $x$-plane from the point $x=2 k$ to the origin. The coefficient $\left(e^{z}-1\right)^{2}$ of $k^{2}$ in equation (1.3) is single-valued and analytic in the cut strip (2.3) and is bounded from zero ${ }^{2}$ there except at the origin.

It is convenient to designate by $S$ that portion of the cut strip (2.3) for which the conditions (2.1) hold. Thus

$$
\begin{aligned}
S=\{z|| \mathfrak{R}(z) \mid \leqq \alpha,-\pi<\mathscr{I}(z) & +\arg k \leqq \pi, \\
& -\pi<\arg z \leqq \pi, \text { and } 0 \leqq \arg k \leqq \pi\} .
\end{aligned}
$$

The part of the strip over which the conditions (2.2) obtain will be designated by $T$. Thus

$$
T=\{z \mid \mathcal{R}(z) \geqq \beta, 0 \leqq \mathscr{S}(z)+\arg k \leqq \pi, \text { and }|\arg k| \leqq \pi\} .
$$

Asymptotic forms of linearly independent solutions of (1.3) will be determined separately over each of the regions $S$ and $T$. The Whittaker functions $W_{k, m}(x)$ and $W_{-k, m}\left(e^{-\pi i} x\right)$ will be first identified with the solutions of (1.3) whose asymptotic forms in $T$ are derived in $\S 3$. The constants $\alpha$ and $\beta$ were deliberately chosen so that $S$ and $T$ overlap so as to be able to connect the solutions whose behavior is derived for $z \varepsilon T$ with those whose behavior is determined in $\$ \$ 5-7$ for $z \varepsilon S$, i.e., near the turning point $z=0$. These connection formulas in turn make possible a description of the asymptotic behavior of the Whittaker functions when the conditions (2.1) are fulfilled. As stated in $\$ 1$, the reasons for separate investigation of (1.3) in $S$ and $T$ are given at the end of $\S 6$.

For future reference it is important to consider the mappings

$$
\xi(z, k)=2 k\left(e^{z}-z-1\right)
$$

and

$$
\zeta(z, k)=\frac{i}{2}\left\{\xi(z, k)+\sigma \ln \left(1-e^{-z}\right)\right\}
$$

of the regions $S$ and $T$, respectively. The image of $T$ with respect to the mapping (2.8) will be called $T_{\zeta}$. The constant $\beta$ is to be chosen so large that $\zeta(z, k) \approx$

\footnotetext{
2 When $\arg k=\pi-\epsilon, \epsilon \geqq 0$ and small, the inequality on $\arg x$ in (2.1) must be replaced by $-\pi+\delta \leqq \arg x \leqq \pi+\delta, \delta>\epsilon$. The analysis changes little in this eventuality, and no further mention will be made of it.
} 
$\frac{1}{2} i x$ for all $z \varepsilon T$. Thus $T_{\zeta}$ consists approximately of the half-plane $\frac{1}{2} \pi \leqq \arg \zeta \leqq \frac{3}{2} \pi$ with a slightly distorted semi-circle of radius $|k| e^{\beta}$ and center at the origin deleted from it. The rays $\arg \zeta=\frac{1}{2} \pi, \frac{3}{2} \pi$ are asymptotes to the boundary of $T_{\zeta}$. It may be seen that through each $z \varepsilon T$ there passes a polygonal path $\Lambda$ joining the points $(\infty, \pi-\arg k),(\Re(z), \pi-\arg k),(\Re(z),-\arg k)$, and $(\infty,-\arg k)$ in order and upon which $\mathfrak{T}(\zeta)$ is strictly monotonic. This property of $T_{\zeta}$ is important with regard to the analysis in $\$ 3$.

The image of $S$ with respect to the mapping (2.7) will be called $S_{\xi}$. It is a portion of a two-sheeted Riemann surface having branch points at zero and infinity. The general features of this mapping are known [7,p. 116]. Since $\alpha>\beta$, $\xi \approx x$ when $R(z)=\alpha$. When $R(z)=-\alpha, \xi \approx-2 k z$. Fig. 2 illustrates the mapping for a typical value of $\arg k\left(\arg k=\frac{1}{4} \pi\right)$.

The portion of $S_{\xi}$ contained in a quadrant $\frac{1}{2} i \pi \leqq \arg \xi \leqq \frac{1}{2}(i+1) \pi, i=-4$, $-3, \cdots, 5$, will be called $Q_{i}$; and its image on $S$ will be called $Q_{i}$. There is a point $\xi_{i}$ of $Q_{i}$ at which $|R(\xi)|$ is a maximum. If there be more than one, let $\xi_{i}$ be the one of this class at which $\mathscr{g}(\xi)$ is a minimum. The corresponding point of $Q_{i}$ will be called $z_{i}$. For each $\xi \varepsilon Q_{i}$ consider the path from 0 to $\xi_{i}$ which consists of those parts of the straight line segments joining 0 to $\xi$ and $\xi$ to $\xi_{i}$ which lie in $\mathcal{Q}_{i}$ plus those portions, if any, of the boundary of $\mathcal{Q}_{i}$ cut off by these segments

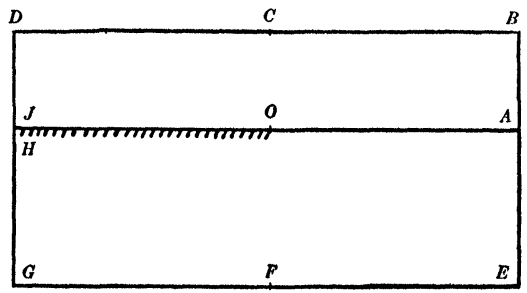

Fig. 2a. The region $S$.

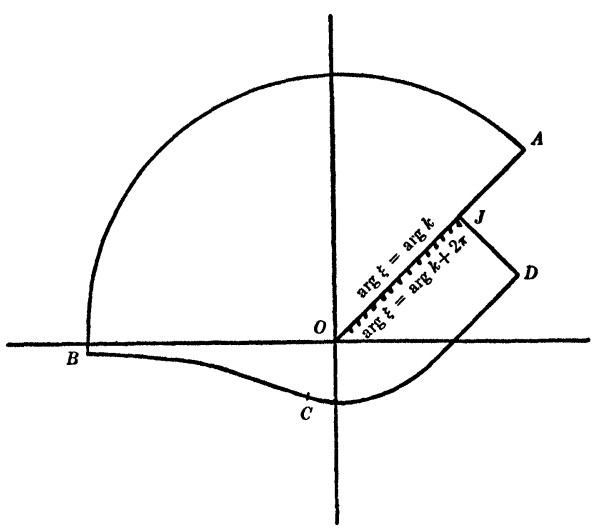

Fig. 2b. $\quad S_{\xi}, \arg k \leqq \arg \xi \leqq \arg k+2 \pi$. 


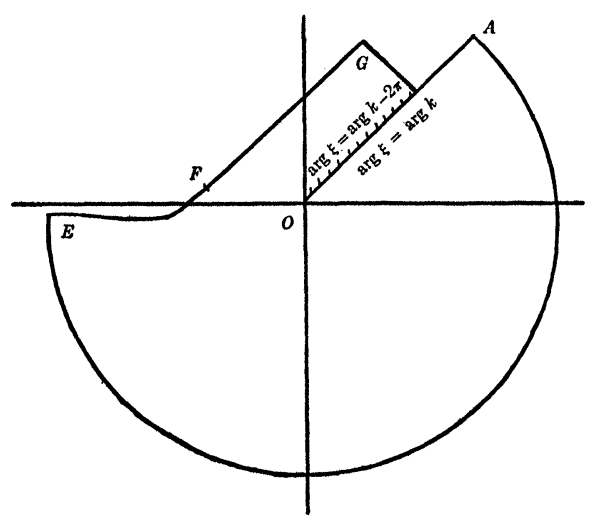

FrG. 2c. $S_{\xi}, \arg k-2 \pi \leqq \arg \xi \leqq \arg k$.

and on which $R(\xi)$ is monotonic. Either of the images in $Q_{i}$ of the two parts of this path joining 0 to $\xi$ and $\xi$ to $\xi_{i}$ will be called a $\Gamma$-curve. The only parts of the boundary of $Q_{i}$ which may have more than one point in common with a $\Gamma$-curve and on which it is not obvious that $R(\xi)$ is monotone are segments of the lines $\mathscr{I}(z)= \pm \pi-\arg k$. When $z=s-i(\arg k \pm \pi), s$ real,

$$
\mathcal{R}(\xi)=-2|k|\left\{e^{*}+(s-1) \cos \arg k+(\sin \arg k)(\arg k \pm \pi)\right\} .
$$

For such $z$ a simple calculation shows that when $R(k) \geqq 0, \mathscr{R}(\xi)$ is a strictly monotonic function of $s$ and when $R(k)<0, \Re(\xi)$ is strictly increasing for $s<\gamma$ and strictly decreasing for $s>\gamma$, where $\gamma=\ln (-\cos \arg k)$. These facts make it clear that $R(\xi)$ is monotone on each $\Gamma$-curve, as stated in the definition above. It is obvious that the $\Gamma$-curves are rectifiable and uniformly bounded in length for all $z \varepsilon S$ and $|k|>N$. The $\Gamma$-curves play an important role in $\$ \S 5$ and 6 .

3. Solutions of (1.3) for $z \varepsilon T$. In the region $T$ defined by (2.6) equation (1.3) has no turning points and hence is an equation of the type considered in $[6, \S 16]$. The theory presented there involves the function $\zeta(z, k)$ defined by $(2.8)$ and functions $\psi(z, k)$ and $\Theta(z, k)$ which for equation (1.3) are found to be

$$
\begin{aligned}
& \psi(z, k)=e^{z}-1+\frac{\sigma}{2 k\left(e^{z}-1\right)}, \\
& \Theta(z, k)=\frac{-\sigma^{2}}{4\left(e^{z}-1\right)^{2}}+\frac{\psi^{\prime \prime}}{2 \psi}-\frac{3 \psi^{2}}{4 \psi^{2}} .
\end{aligned}
$$

If $|k|>N, \psi(z, k)$ is bounded from zero in $T$. When $R(z)>N, \psi, \psi^{\prime}$, and $\psi^{\prime \prime}$ are all nearly equal to $e^{z}$ so that $|\Theta(z, k)|<M$, when $z \varepsilon T$ and $|k|>N$. Thus the integral

$$
\int_{\Delta} \frac{\Theta(s, k)}{\psi(s, k)} d s
$$


taken along a $\Lambda$-curve joining $(\infty,-\arg k)$ or $(\infty, \pi-\arg k)$ to $z$ is uniformly bounded for $z \varepsilon T$ and $|k|>N$.

The foregoing remarks together with the properties of $T_{\zeta}$ noted in $\S 2$ establish the fact that the hypotheses set forth in $[6, \S 16]$ are satisfied by the differential equation (1.3) in $T$. Therefore there exist linearly independent solutions $U_{1}(z, k)$ and $U_{2}(z, k)$ of this equation which for all $z \varepsilon T$ and $|k|>N$ have the asymptotic forms

$$
\begin{aligned}
& U_{1}(z, k)=\psi^{-\frac{1}{2}}(z, k) e^{i \zeta(z, k)}\left[1+\frac{E(z, k)}{k}\right], \\
& U_{2}(z, k)=\psi^{-\frac{1}{2}}(z, k) e^{-i \zeta(z, k)}\left[1+\frac{E(z, k)}{k}\right] .
\end{aligned}
$$

The asymptotic behavior of $W_{k, m}(x)$ and $W_{-k, m}\left(e^{-\pi i} x\right)$ subject to the conditions (2.2) may now be identified by comparing the behavior at $x=\infty$ of the solutions $e^{-\frac{1}{2} z} W_{k, m}\left(2 k e^{z}\right)$ and $e^{-\frac{1}{2} z} W_{-k, m}\left(2 k e^{2-\pi i}\right)$ of (1.3) with that of the linearly independent solutions $U_{1}(z, k)$ and $U_{2}(z, k)$. The identities relating these solutions may be written as

$$
\begin{aligned}
\text { (a) } e^{-\frac{1}{2} z} W_{k, m}\left(2 k e^{z}\right) & =C_{1,1} U_{1}(z, k)+C_{1,2} U_{2}(z, k) \\
\text { (b) } e^{-\frac{1 z}{z}} W_{-k, m}\left(2 k e^{z-\pi i}\right) & =C_{2,1} U_{1}(z, k)+C_{2,2} U_{2}(z, k)
\end{aligned}
$$

in which the coefficients $C_{h, i}$ are independent of $z$ but possibly dependent upon the parameters $k$ and $m$. It may be seen that

$$
\psi(z, k)=\left(\frac{x}{2 k}-1\right)+\frac{\sigma}{x-2 k}, \quad e^{i \zeta(z, k)}=\left(\frac{x}{2 k}\right)^{k+\frac{1}{2} \sigma}\left(\frac{x}{2 k}-1\right)^{-\frac{1}{2} \sigma} e^{k-\frac{1}{2} x} .
$$

If $z \varepsilon T$, then $U_{1}(z, k)$ and $U_{2}(z, k)$ may be replaced in (3.3a) by their asymptotic forms (3.2), and $\psi$ and $e^{i \zeta}$ may be replaced in turn by their equivalents (3.4). The identity (3.3a) may then be given the form

$$
\begin{aligned}
& e^{\frac{1}{x} x} x^{-k} W_{k, m}(x)=C_{1,1}\left(\frac{e}{2 k}\right)^{k}(\left.1-\frac{2 k}{x}\right)^{-\frac{1}{2}(\sigma+1)}\left[1+\frac{E(z, k)}{k}\right] \\
&+C_{1,2}\left(\frac{2 k}{e x^{2}}\right)^{k}\left(1-\frac{2 k}{x}\right)^{\frac{1}{2}(\sigma-1)} e^{x}\left[1+\frac{E(z, k)}{k}\right] .
\end{aligned}
$$

Now, the function $W_{k, m}(x)$ is known to have the property

$$
W_{k, m}(x)=e^{-\frac{1}{2} x} x^{k}\left[1+\frac{E(x)}{x}\right], \quad|\arg x|<\frac{3}{2} \pi, \quad|x|>N .
$$

Thus, since (3.5) holds even if $R(x) \rightarrow+\infty$, it must be that

$$
C_{1,2}=0 ; \quad \text { and hence, } \quad C_{1,1}=\left(\frac{2 k}{e}\right)^{k}\left[1+\frac{E(k)}{k}\right] \text {. }
$$

The coefficients $C_{2,1}$ and $C_{2,2}$ in the identity (3.3b) may be evaluated similarly 
by letting $R(x) \rightarrow-\infty$. Their values are

$$
C_{2,1}=0, \quad C_{2,2}=\left(\frac{e^{\pi i+1}}{2 k}\right)^{k}\left[1+\frac{E(k)}{k}\right] .
$$

These results may be summarized in the following

Theorem 1. When $|k|>N,|\sigma|<M$, and the conditions (2.2) hold,

$$
W_{k, m}(x)=\left(1-\frac{2 k}{x}\right)^{-\frac{1}{2}(\sigma+1)} e^{-\frac{3}{3} x} x^{k}\left[1+\frac{E(x, k)}{k}\right]
$$

(b) $W_{-k, m}\left(e^{-\pi i} x\right)=\left(1-\frac{2 k}{x}\right)^{\frac{1}{2}(\sigma-1)} e^{k \pi i+\frac{1}{3} x} x^{-k}\left[1+\frac{E(x, k)}{k}\right]$,

where $\sigma=\left(m^{2}-k^{2}\right) / k$. The $E$ symbols denote functions uniformly bounded in $x$ and $k$ when the number $\beta$ in (2.2) and $N$ are sufficiently large.

If $-\pi \leqq \arg k \leqq-\epsilon<0$, then equation (1.3) has no turning point for $0 \leqq \arg x \leqq \pi$. It thus becomes possible to partially extend the results of Theorem 1 to the region $|x / 2 k| \geqq e^{-\beta}, 0 \leqq \arg x \leqq \pi$. A full extension is not possible because the enlarged $T_{5}$ fails to fulfill the hypothesis (b) in [6, §16]. The limited extension is

Theorem 2. When $|x / 2 k| \geqq e^{-\beta}, \beta>0$, and $0 \leqq \arg x \leqq \pi$, the representations (3.9a) and (3.9b) hold if $-\pi \leqq \arg k \leqq-\frac{1}{2} \pi$ and $-\frac{1}{2} \pi \leqq \arg k \leqq-\epsilon<0$, respectively.

This theorem gives the asymptotic form of only one solution of the Whittaker equation (1.1) for each admissible $x$.

Asymptotic forms for the functions $M_{k, m}(x)$ and $M_{k,-m}(x)$ can be found by substitution from (3.9) into the familiar relation

$$
M_{k, m}(x)=\Gamma(2 m+1)\left\{\frac{e^{-k \pi i} W_{-k, m}\left(e^{-\pi i} x\right)}{\Gamma\left(m-k+\frac{1}{2}\right)}+\frac{e^{\left(m-k+\frac{1}{2}\right) \pi i} W_{k, m}(x)}{\Gamma\left(m+k+\frac{1}{2}\right)}\right\} .
$$

It can be seen that the relations (3.9) are consistent with Kummer's transformation of $M_{k, m}(x)$. Theorem 1 is also in accord with the fact that $W_{k, k-\frac{1}{2}}(x)=$ $x^{k} e^{-\frac{1}{2} x}$. Finally, it may be noted that the behavior of $W_{k, m}(x)$ and $W_{-k, m}\left(e^{-\pi i} x\right)$ when $k$ and $m$ are both bounded and $|x|$ is large is to a first approximation the same as that exhibited by them in Theorem 1 .

4. A related equation. The next task is to determine the asymptotic forms of solutions of the transformed Whittaker equation (1.3) for $z \varepsilon S$. To accomplish this MCKeLvey's second order turning point theory [9] will be extended to the complex domain, but only for the particular equation (1.3). The first step is to derive a related equation of the form

$$
\frac{d^{2} u}{d z^{2}}-\left\{k^{2}\left(e^{z}-1\right)^{2}+k \sigma+E(z, k)\right\} u=0,
$$


the asymptotic behavior of whose solutions for large $|k|$ is known. The function designated above by $E$ is bounded for $z \varepsilon S$ and $|k|>N$. It will be seen that the solutions of (4.1) are related to the parabolic cylinder functions. These are solutions of WEBER's equation which is the simplest second order differential equation with two first order transition points. The derivation to be made is a specialization of the analogous step in [9], and the relations contained in this section are in most cases specializations of more general ones in [9]. The motivation of the choice of (4.1) is not repeated here.

The differential equation

$$
\frac{d^{2} v}{d z^{2}}-R(z, k) v=0
$$

in which

$$
\begin{aligned}
\text { (a) } R(z, k) & =k^{2} \varphi^{2}(z)+\frac{k \sigma}{2 \Psi^{4}(z)}+\frac{\Psi^{\prime \prime}(z)}{\Psi(z)} \\
\text { (b) } \varphi(z) & =e^{z}-1 \\
\text { (c) } \Psi(z) & =\frac{\left(e^{z}-z-1\right)^{\frac{1}{2}}}{\left(e^{z}-1\right)^{\frac{3}{2}}}, \quad z \neq 0, \text { and } \Psi(0)=2^{-\frac{1}{4}}
\end{aligned}
$$

is satisfied by any function of the form

$$
v(z, k)=\Psi(z) \xi^{-\frac{1}{2}} M(\xi),
$$

where $M(\xi)$ is any linear combination of the Whittaker functions $W_{-\frac{1}{2} \sigma, \frac{1}{2}}(\xi)$ or $M_{-\sigma_{\frac{1}{2}}, \pm \frac{1}{2}}(\xi)$. The function $\xi(z, k)$ is defined by $(2.7)$.

When $|\xi| \leqq N$, it is convenient to use the solutions ${ }^{3}$

$$
v_{j}(z, k)=\Psi(z) \xi^{-\frac{1}{2}} M_{-\frac{2}{2} \sigma, j}(\xi), \quad j= \pm \frac{1}{4} .
$$

When $|\xi|>N$, the solutions ${ }^{4}$

$$
v_{\nu}(z, k)=\Psi(z) \xi^{-\frac{1}{2}} W_{\nu}(\xi),
$$

wherein

$$
W_{\nu}(\xi)=W_{-\bar{z} \sigma, \frac{1}{2}}\left(e^{-\nu \pi i} \xi\right), \quad \nu=0, \pm 1, \pm 2,
$$

are to be used because of the simplicity of their asymptotic forms. Let

$$
\begin{aligned}
\Xi_{\nu}=\left\{\xi \mid \xi \varepsilon S_{\xi} \text { and }\left(\nu-\frac{1}{2}\right) \pi+\epsilon \leqq \arg \xi \leqq\left(\nu+\frac{3}{2}\right) \pi-\epsilon,\right. & \\
& \nu=0, \pm 1, \pm 2\} .
\end{aligned}
$$

\footnotetext{
${ }^{3}$ In this and succeeding formulas containing both the index $j$ and the symbols \pm or $\mp$ the upper sign is to be used when $j=\frac{1}{4}$, the lower when $j=-\frac{1}{4}$.

${ }^{4}$ When the index $\nu$ appears in a formula together with the symbols \pm or $\mp$, the upper sign is to be used when $\nu$ is even, the lower when $\nu$ is odd.
} 
For $\xi \varepsilon \Xi_{\nu}$, the asymptotic representations of $W_{\nu}(\xi)$ and $W_{v+1}(\xi)$ given by (3.6) both hold. These representations may be differentiated with respect to $\xi$ when $\xi \varepsilon \Xi_{\nu}$.

With

(a)

$$
\theta(z)=\frac{\sigma}{4} \ln \frac{\left(e^{z}-1\right)^{2}}{2\left(e^{z}-z-1\right)}-\frac{\sigma z}{2}
$$

(b) $\quad \mu_{0}(z)=\cosh \theta, \quad \mu_{1}(z)=(\sinh \theta) / \varphi(z), \quad z \neq 0, \quad \mu_{1}(0)=-\sigma$

$$
D_{0}(z, k)=\left|\begin{array}{cc}
\mu_{0} & \mu_{0}^{\prime}+\mu_{1} R / k \\
\mu_{1} / k & \mu_{0}+\mu_{1}^{\prime} / k
\end{array}\right|
$$

the functions

$$
y(z, k)=D_{0}^{-\frac{1}{2}}(z, k)\left[\mu_{0}(z) v(z, k)+\frac{\mu_{1}(z) v^{\prime}(z, k)}{k}\right]
$$

satisfy a related equation of the form (4.1). The bounded function represented by $E$ is not given explicitly as its specific value is not needed in the sequel. When $z \varepsilon S$ and $|k|>N, D_{0}(z, k)=1+E(z, k) / k$; and therefore the division by $D_{0}^{\frac{1}{2}}(z, k)$ in (4.10) is permissible. It may be said that the function $E(z, k)$ appearing in (4.1) is sufficiently complicated to prevent, for practical purposes, a better approximation to (1.3) than (4.1). In other words, the quadratures in $[9, \S 4]$ cannot be carried out explicitly. The approximation is, however, close enough for solutions of (1.3) to resemble solutions of (4.1). This is not altogether obvious since it might be expected that a discussion based upon the use of (4.1) as a related equation would lead to the conclusions of the theorems in [9] with $n=-1$, in which case the error terms would be too large and the conclusions useless.

Through the relation (4.10) there correspond to the various solutions (4.5) and (4.6) of (4.2) solutions of the related equation which will be denoted $y_{j}(z, k)$ and $y_{\nu}(z, k)$. When $|\xi|>M,|z|<M|k|^{-\frac{1}{2}}$. Therefore, when $|\xi| \leqq M$ and $|k|>N$, a computation shows that

$$
\begin{array}{ll}
\text { (a) } \quad y_{j}(z, k)=E(z, k), & y_{i}^{\prime}(z, k)=k^{\frac{1}{3}} E(z, k) \\
\text { (b) } \quad y_{v}(z, k)=E(z, k), & y_{v}^{\prime}(z, k)=k^{\frac{1}{3}} E(z, k) .
\end{array}
$$

The Wronskian

$$
\left|\begin{array}{ll}
f_{1}(z) & f_{2}(z) \\
f_{1}^{\prime}(z) & f_{2}^{\prime}(z)
\end{array}\right|
$$

will be abbreviated by $w\left(f_{1}, f_{2}, z\right)$. Since

$$
\mathscr{W}\left(y_{i}, y_{-i}, z\right)=\mp(k / 2)^{\frac{1}{2}} \text { and } W\left(y_{v}, y_{v+1}, z\right)= \pm e^{-\frac{1}{z} \sigma \pi i}(2 k)^{\frac{1}{2}}
$$

are not zero, both $\left(y_{2}, y_{-\frac{1}{2}}\right)$ and $\left(y_{v}, y_{v+1}\right)$ are pairs of linearly independent solutions of the related equation. The solutions $y_{i}$ and $y_{v}$ are, of course, not all linearly independent. The relation 


$$
W_{\nu}(\xi)=\frac{e^{-\frac{1}{2} \nu \pi i} \Gamma\left(-\frac{1}{2}\right)}{\Gamma\left(\frac{1 \pm \sigma}{4}\right)} M_{-\frac{1}{2} \sigma, \frac{1}{4}(\xi)}+\frac{e^{-\frac{1}{2} \nu \pi i} \Gamma\left(\frac{1}{2}\right)}{\Gamma\left(\frac{3 \pm \sigma}{4}\right)} M_{-\frac{1}{2} \sigma,-\frac{1}{2}(\xi)}
$$

is known [2, pp. 18 and 26]. From it the inverse relations

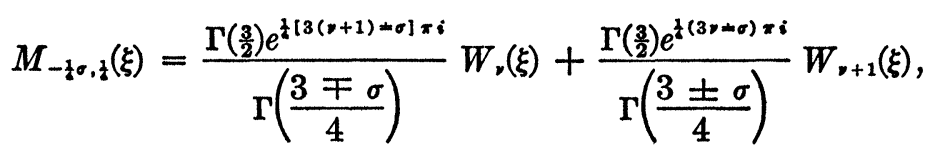

$$
\begin{aligned}
& M_{-\frac{1}{2} \sigma,-\frac{1}{2}(\xi)}=\frac{\Gamma\left(\frac{1}{2}\right) e^{\frac{z}{\xi(\nu+1+\sigma) \pi i}}}{\Gamma\left(\frac{1 \mp \sigma}{4}\right)} W_{\nu}(\xi)+\frac{\Gamma\left(\frac{1}{2}\right) e^{z(\nu+\sigma) \pi i}}{\Gamma\left(\frac{1 \pm \sigma}{4}\right)} W_{v+1}(\xi)
\end{aligned}
$$

may be derived. If $\sigma$ is such that the argument of any of the gamma functions occurring in these formulas is zero or a negative integer, the term in which it appears is to be omitted. The relations of linear dependence among the solutions $y_{i}$ and $y_{v}$ may easily be found on the basis of (4.13) and (4.14).

There will also be occasion to use the relations

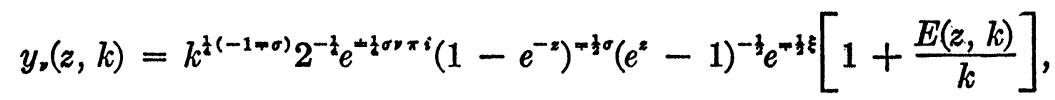

$$
\begin{aligned}
& y_{v+1}(z, k)=k^{z(-1+\sigma)} 2^{-\frac{1}{z}} e^{-z \sigma(v+1) \pi i}\left(1-e^{-z}\right)^{-\frac{1}{z} \sigma}\left(e^{z}-1\right)^{-\frac{k}{z}} e^{-z \xi}\left[1+\frac{E(z, k)}{k}\right],
\end{aligned}
$$

which may be derived from (3.6), (4.6), and (4.10) when $|\xi|>N$ and $\xi \varepsilon \Xi_{y}$. In these formulas the first derivatives of the functions designated by $E(z, k)$ are also bounded in $z$ and $k$. Consequently, the asymptotic forms for $y_{r}^{\prime}$ and $y_{r+1}^{\prime}$ when $\xi \varepsilon \Xi_{\nu}$ are the derivatives of those for $y_{\nu}$ and $y_{\nu+1}$, respectively.

5. Solutions $u_{i}(z, k)$ when $z \varepsilon S$ and $|\xi(z, k)| \leqq M$. It will next be shown that solutions of the related equation are asymptotic representations of the solutions of (1.3). The following lemma will be useful. For a proof see [8].

Lemma. If (a) $A$ is a bounded simply connected region whose boundary may depend upon a complex parameter $\lambda,(b) f(z, \lambda)$ is continuous and bounded for $z \varepsilon A$ and $|\lambda|>N,(c) H(z, t, \lambda)$ is continuous for $z \varepsilon A$ and $t \varepsilon A$, and $(d)$ for $s \varepsilon A$ independent of $z$, all $z$ in $A,|\lambda|>N$, and a set of rectifiable paths joining points of $A$ to $s$

$$
\int_{.}^{z}|H(z, t, \lambda)||d t|<M
$$

then the solution $g(z, \lambda)$ of the integral equation

$$
g(z, \lambda)=f(z, \lambda)+h(\lambda) \int_{0}^{*} H(z, t, \lambda) g(t, \lambda) d t,
$$

where $h(\lambda)=\lambda^{-1}$ or $\lambda^{-1} \ln \lambda$, is of the form

$$
g(z, \lambda)=f(z, \lambda)+h(\lambda) E(z, \lambda)
$$


for $|\lambda|>N$ and $z \varepsilon A$.

A familiar precedure shows that (1.3) is equivalent to a Volterra equation of the form

$$
u(z)=y(z)+\int_{z_{*}}^{z}\left[y_{a}(z) y_{b}(t)-y_{b}(z) y_{a}(t)\right] \frac{E(t, k) u(t)}{\mathscr{W}\left(y_{a}, y_{b}, t\right)} d t,
$$

in which $y_{a}$ and $y_{b}$ are any two linearly independent solutions of the related equation, $y$ is any solution, and both $z$ and $z_{*}$ are points of the region $S$ defined by (2.5). Inasmuch as the integrand in (5.1) is analytic in $S$, the path of integration may be chosen at pleasure so as to facilitate the appraisal of solutions of this equation when $|k|$ is large. The reader should recall in this connection the definition of a $\Gamma$-curve made in the last paragraph of $\$ 2$.

The integral equation satisfied by $u^{\prime}(z, k)$ is

$$
u^{\prime}(z)=y^{\prime}(z)+\int_{z_{*}}^{z}\left[y_{a}^{\prime}(z) y_{b}(t)-y_{b}^{\prime}(z) y_{a}(t)\right] \frac{E(t, k) u(t)}{W\left(y_{a}, y_{b}, t\right)} d t .
$$

When $|\xi(z, k)| \leqq M$, the asymptotic forms of solutions of (5.1) are easily found. In (5.1) choose $z_{*}=0, y=y_{i}, y_{a}=y_{k}$, and $y_{b}=y_{-\frac{1}{k}}$. These choices determine a solution $u_{i}(z, k)$ of (5.1) such that

$$
u_{i}(0, k)=y_{i}(0, k) \text { and } u_{i}^{\prime}(0, k)=y_{i}^{\prime}(0, k) .
$$

Let $B=\{z \mid z \varepsilon S$ and $|\xi(z, k)| \leqq M\}$. The boundary of $B$, of course, depends upon $k$. With $z_{*}=0$ and $z \varepsilon B$ the path of integration in (5.1) may be chosen to be the arc of the $\Gamma$-curve directed from 0 to $z$, provided it lies entirely in $B$. If it does not, replace that part of it not in $B$ by appropriate arcs of the boundary of $B$ so that there will be a continuous integration path lying in $B$. By referring to the relations (4.11) and (4.12) and also noting that

$$
d t=\frac{\Psi^{2}(t) d \xi}{(2 k)^{\frac{1}{3}} \xi^{\frac{1}{2}}(t)}
$$

it may be deduced that in these circumstances the integral of the kernel in (5.1) is bounded by $M /|k|$. Thus, when $A=B, f=y_{i}, s=0, \lambda=k$, and $h(k)=k^{-1}$, the hypotheses of the lemma are seen to be fulfilled with respect to equation (5.1). Therefore, when $z \varepsilon B$ and $|k|>N$,

$$
u_{i}(z, k)=y_{i}(z, k)+\frac{E(z, k)}{k}, \quad j= \pm \frac{1}{4} .
$$

It now follows directly from (5.2), (4.11), and (5.4) that

$$
u_{i}^{\prime}(z, k)=y_{i}^{\prime}(z, k)+\frac{E(z, k)}{k^{3}}
$$

for $z \varepsilon B$ and $|k|>N$. 
6. Solutions $u_{\mu}(z, k)$ for unrestricted $|\xi|$ when $\xi \varepsilon Q_{i}$. Let it now be assumed that $\xi \varepsilon Q_{i} \subset \Xi_{\nu}$, where $\nu$ is odd. This restricts $z$ to the bounded simply connected subregion $Q_{i}$ of $S$. The reader should recall, at this point, the definitions of $Q_{i}, Q_{i}$, and $z_{i}$ made in $\S 2$. The choices $y=y_{\nu}, y_{a}=y_{\nu}, y_{b}=y_{\nu+1}$, and $z_{*}=0$ if $R(\xi) \geqq 0$ for $\xi \varepsilon Q_{i}$ but $z_{*}=z_{i}$ if $R(\xi) \leqq 0$ for $\xi \varepsilon Q_{i}$ determine a solution $u_{\nu}(z, k)$ of (5.1). The kernel of this specialization of (5.1) will be called $k^{-\frac{1}{2}} K(z, t, k)$. It is necessary to discuss two cases in determining the asymptotic behavior of $u_{\nu}(z, k)$.

I. $R(\xi) \geqq 0$ in $Q_{i}$. Let $B_{1}=\left\{z \mid z \varepsilon Q_{i}\right.$ and $\left.|\xi(z, k)| \leqq M\right\}$ and $B_{2}=\left\{z \mid z \varepsilon Q_{i}\right.$ and $|\xi(z, k)| \geqq M\}$. Since $R(\xi) \geqq 0$ in $Q_{i}$, the path of integration in (5.1) is from the origin to $z$; and therefore, if $z \varepsilon B_{1}$, the reasoning leading to (5.4) may be repeated to show that

$$
u_{\nu}(z, k)=y_{\nu}(z, k)+\frac{E(z, k)}{k}=E(z, k)
$$

if $z \varepsilon B_{1}$ and $|k|>N$.

If $z \varepsilon B_{2}$, the path of integration is chosen as follows. On the $\Gamma$-curve directed from the origin to $z$ there is a first point $z_{a}$ and a last point $z_{b}$ in $B_{1} \cap B_{2}$. These points may, of course, coincide. If there is a unique point of $B_{1} \cap B_{2}$ at which $R(z)$ is a maximum, call it $z_{+}$. If there is more than one such point, let $z_{+}$be the one of this class at which $\mathscr{g}(z)$ is a maximum. The path of integration is to consist of the $\Gamma$-curve directed from 0 to $z_{a}$, the portion of $B_{1} \cap B_{2}$ joining $z_{a}$ to $z_{+}$, the portion of $B_{1} \cap B_{2}$ joining $z_{+}$to $z_{b}$, and the segment from $z_{b}$ to $z$ of the $\Gamma$-curve joining the origin to $z$.

On the part of the integration path from 0 to $z_{+}, t \varepsilon B_{1}$, so that $u_{\nu}(t, k)=$ $E(t, k)$. It follows by a simple computation using (4.11b) that

$$
k^{-\frac{1}{2}} \int_{0}^{z_{+}} K(z, t, k) u_{\nu}(t, k) d t=y_{\nu}(z, k) k^{-1} E(z, k) .
$$

Equation (5.1) may now be written in the form

where

$$
\begin{aligned}
u_{\nu}(z) \Delta(\xi, z)=y_{\nu}(z) \Delta(\xi, z) & {\left[1+\frac{E(z, k)}{k}\right] } \\
+ & k^{-\frac{1}{2}} \int_{z_{+}}^{z}\left\{\xi^{\frac{1}{2}}(t)\left[\frac{\Delta(\xi, z)}{\Delta(\xi, t)}\right] K(z, t, k)\right\} u_{\nu}(t) \Delta(\xi, t) \frac{d t}{\xi^{\frac{3}{2}}(t)}
\end{aligned}
$$

$$
\Delta(\xi, z)=e^{-\frac{1}{2} \xi(z)} \xi^{\frac{1}{4}(1-\sigma)} .
$$

The divisions made in transforming (5.1) to (6.2) are legitimate as both $|\xi(z)|$ and $|\xi(t)|$ are large. The part of the integrand in (6.2) enclosed by curly brackets is bounded on the integration path from $z_{+}$to $z_{b}$, where $|\xi(t)|=M$; and since $\Re(\xi(t))<R(\xi(z))$ on the path from $z_{b}$ to $z$, it is also bounded on this last segment from $z_{b}$ to $z$. Moreover,

$$
\left|k^{-\frac{1}{2}} \int_{z+}^{z} \frac{d t}{\xi^{\frac{1}{2}}(t)}\right|<\frac{M}{4|k|}\left\{\int_{\xi(z+)}^{\xi\left(z_{b}\right)} \frac{|d \xi|}{|\xi|}+\int_{\xi_{(z b)}}^{\xi(z)} \frac{|d \xi|}{|\xi|}\right\}<\frac{M \ln |k|}{|k|} .
$$


As a consequence of these facts, the hypotheses of the lemma may be seen to be fulfilled with respect to equation (6.2) when $A=B_{2}, \lambda=k, f(z, k)=$ $y_{\nu}(z, k) \Delta(\xi, z), g(z, k)=u_{\nu}(z, k) \Delta(\xi, z), s=z_{+}$, and $h(k)=k^{-1} \ln k$. The lemma therefore justifies the conclusion that when $z \varepsilon B_{2}$ and $|k|>N$,

$$
u_{\nu}(z, k)=y_{\nu}(z, k)+\frac{E(z, k) \ln k}{\Delta(\xi, z) k}=y_{\nu}(z, k)\left[1+\frac{E(z, k) \ln k}{k}\right] .
$$

It follows from (6.3), (6.1), (4.15), (4.11b), and (5.2) that the form of $u_{\nu}^{\prime}(z, k)$ for $|k|>N$ and $z$ in either $B_{1}$ or $B_{2}$ may be obtained from the corresponding form of $u_{\nu}(z, k)$ by replacing $y_{\nu}(z, k)$ by $y_{\nu}^{\prime}(z, k)$ and $E(z, k) / k$ by $E(z, k) / k^{\frac{1}{2}}$.

II. $\Omega(\xi) \leqq 0$ in $Q_{i}$. If $z \varepsilon B_{2}$, one may show by an argument like that in the last paragraph that the representation (6.3) again holds for $z \varepsilon B_{2}$ and $|k|>N$. If $z \varepsilon B_{1}$, the integration path is to consist of (a) the segment joining $z_{i}$ to $z_{b}$ of the $\Gamma$-curve directed from $z_{i}$ to $z_{+}$, where $z_{b}$ is the first point in $B_{1}$ on the $\Gamma$-curve, (b) the arc of $B_{1} \cap B_{2}$ from $z_{b}$ to $z_{+}$, and (c) a curve lying in $B_{1}$ whose image in $Q_{i}$ is a polygonal path joining $\xi\left(z_{+}\right)$to $\xi(z)$ and is made up of the smallest possible number of straight line segments. These paths are clearly uniformly bounded in length for $z \varepsilon Q_{i}$.

On the portion of the path from $z_{i}$ to $z_{+}, t \varepsilon B_{2}$, so that $u_{\nu}(t, k)=y_{\nu}(t, k) \cdot$ $\left[1+E(t, k) k^{-1} \ln k\right]$. The functions $y_{\nu}(z)$ and $y_{\nu+1}(z)$ are bounded for $z \varepsilon B_{1}$ and $|k|>N$. From (4.15) and the hypothesis that $R(\xi) \leqq 0$ in $Q_{i}$ it follows that on this part of the path $\xi^{\frac{1}{2}}(t) y_{\nu}(t) y_{\nu+1}(t)$ and $\xi^{\frac{1}{2}}(t) y_{\nu}^{2}(t)$ are bounded. Consequently,

$$
k^{-\frac{1}{2}} \int_{z_{i}}^{z+} K(z, t, k) u_{\nu}(t, k) d t=E(z, k) k^{-1} \ln k
$$

for $z \varepsilon B_{1}$ and $|k|>N$. Knowing this, one may now write the integral equation (5.1) in the form

$$
u_{\nu}(z, k)=y_{\nu}(z, k)+\frac{E(z, k) \ln k}{k}+k^{-\frac{1}{2}} \int_{z+}^{z} K(z, t, k) u(t, k) d t .
$$

The kernel in (6.4) is bounded on the integration path from $z_{+}$to $z$ as both $t$ and $z$ lie in $B_{1}$; hence, it follows from the lemma that the representation (6.1) again obtains for $z \varepsilon B_{1}$ and $|k|>N$. The appraisal of $u_{\nu}^{\prime}(z, k)$ may be carried out as in Case I. This completes the discussion of Case II.

The preceding results do not involve any restrictions upon arg $\sigma$. In both this respect and in the error estimates the results improve upon those given in [9]. Modified versions of the arguments given in the discussion of Cases I and II lead to analogous results in the event that $\nu$ is even. The form of a second solution $u_{\nu+1}(z, k)$ and its derivative may thus be derived for each $\nu$. It is therefore possible to prove the following:

Theorem 3. Corresponding to each subregion $Q_{i}$ contained in a sector $\Xi_{\nu}$ defined' by (4.8) there exists a pair of linearly independent solutions $u_{\nu}(z, k)$ and $u_{p+1}(z, k)$ of the transformed Whittaker equation (1.3) such that when $|\xi(z, k)| \leqq M$ and $|k|>N$, 


$$
\begin{aligned}
& u_{\mu}(z, k)=y_{\mu}(z, k)+\frac{E(z, k) \ln k}{k} \\
& u_{\mu}^{\prime}(z, k)=y_{\mu}^{\prime}(z, k)+\frac{E(z, k) \ln k}{k^{\ddagger}},
\end{aligned}
$$

and when $|\xi(z, k)| \geqq M$ and $|k|>N$,

$$
\begin{aligned}
& u_{\mu}(z, k)=y_{\mu}(z, k)\left[1+\frac{E(z, k) \ln k}{k}\right] \\
& u_{\mu}^{\prime}(z, k)=y_{\mu}^{\prime}(z, k)\left[1+\frac{E(z, k) \ln k}{k^{3}}\right]
\end{aligned}
$$

where $\mu=\nu, \nu+1$ and $\nu=0, \pm 1, \pm 2$.

The solutions designated with the same subscript $\mu$ are, in general, different for distinct subregions $\mathcal{Q}_{i}$ of the same sector $\Xi_{\nu}$. The conclusions of Theorem 3 may, however, be strengthened when $\nu=0$. The key facts which permit this are that all points of $S$ for which $-\frac{3}{2} \pi \leqq \arg \xi(z) \leqq \frac{3}{2} \pi\left(-\frac{1}{2} \pi \leqq \arg \xi(z) \leqq \frac{3}{2} \pi\right)$ are accessible from $z_{0}\left(z_{1}\right)$ by paths which, as seen on $S_{\xi}$, are made up of a finite number of straight line segments and on which $R(\xi)$ is monotonic decreasing (increasing). The points $z_{0}$ and $z_{1}$ are those defined in §2. Arguments like those used in the preceding discussions may thus be used to show that the solution $u_{0}(z, k)$ of (5.1) defined by the choices $y=y_{0}, y_{a}=y_{0}, y_{b}=y_{1}$, and $z_{*}=z_{0}$ possesses the representations (6.5) and (6.6) for all $z \varepsilon S$ such that $-\frac{1}{2} \pi \leqq \arg$ $\xi(z) \leqq \frac{3}{2} \pi$. Similarly, there is a solution $u_{1}(z, k)$ of $(5.1)$ corresponding to the choices $y=y_{1}, y_{a}=y_{0}, y_{b}=y_{1}$, and $z_{*}=z_{1}$ which possesses the representations (6.5) and (6.6) for all $z \varepsilon S$ such that $-\frac{1}{2} \pi \leqq \arg \xi(z) \leqq \frac{3}{2} \pi$. This extension of Theorem 3 with respect to $u_{0}$ and $u_{1}$ proves to be useful in identifying the behavior of the Whittaker functions $W_{k, m}(x)$ and $W_{-k, m}\left(e^{-\pi i} x\right)$ when $z(x, k)$ is in $S$.

It may now be explained why the investigation of solutions of (1.3) made in $\S \S 4,5$, and 6 cannot be extended to the unbounded region $S \cup T$. The basic difficulty appears to stem from the presence of the linear term in $k$ in the coefficient of $u$ in (1.3). The method of investigation requires the boundedness of $K(z, t, k)$ or its integral over suitable paths. When $z$ is restricted to $S$, the functions $\mu_{0}(z)$ and $\mu_{1}(z)$ defined by $(4.9 \mathrm{~b})$ and which are involved in the functions $y_{v}(z, k)$, and therefore in $K(z, t, k)$, are bounded. However, if $R(z) \rightarrow+\infty, \mu_{0}(z)$ and $\mu_{1}(z)$ are in general not bounded. This fact is reflected in the behavior of $K(z, t, k)$ and has caused difficulties which the author has been unable to surmount except by the means of circumventing the problem with the analysis given in §3. The same sort of difficulty occurs in [6], again in the presence of a linear term in the large parameter.

7. Connection formulas. The connection between the solutions $u_{i}(z, k)$ of $\S 5$ and the solutions $u_{\mu}(z, k)$ of $\S 6$ will now be established. To accomplish this it is 
necessary only to evaluate the coefficients in the linear relations

(a) $u_{\mu}(z, k)=\alpha_{1, \mu}(k) u_{2}(z, k)+\alpha_{2, \mu}(k) u_{-\frac{1}{2}}(z, k), \quad \mu=\nu, \nu+1$

(b) $u_{i}(z, k)=\beta_{i, \nu}(k) u_{\nu}(z, k)+\beta_{i, v+1}(k) u_{\nu+1}(z, k), \quad j= \pm \frac{1}{4}$.

A sample calculation is

$$
\alpha_{2, \mu}(k)=\frac{\mathscr{W}\left(u_{\frac{1}{2}}, u_{\mu}, z\right)}{\mathscr{W}\left(u_{\frac{1}{2}}, u_{-\frac{1}{2}}, z\right)}
$$

or by (5.3) and (6.5)

$$
\alpha_{2, \mu}(k)=\frac{W\left(y_{\frac{1}{2}}, y_{\mu}, 0\right)}{W\left(y_{\frac{1}{2}}, y_{-\frac{1}{2}}, 0\right)}\left[1+\frac{E(k) \ln k}{k}\right] .
$$

Because the formula (4.10) is linear in $v$ and $v^{\prime}$, the quotient of the Wronskians in the last expression is equal to the coefficient of $M_{-\frac{1}{2} \sigma,-\frac{1}{2}}(\xi)$ in (4.13); see [9, p. 109]. In evaluating the $\beta$ 's the identities (4.14) are used. The conclusions $\operatorname{are}^{5}$ :

$$
\begin{aligned}
\alpha_{1, \mu}(k) & =\frac{e^{-\frac{3}{2 \mu \pi i}} \Gamma\left(-\frac{1}{2}\right)}{\Gamma\left(\frac{1 \pm \sigma}{4}\right)}\left[1+\frac{E(k) \ln k}{k}\right] \\
\alpha_{2, \mu}(k) & =\frac{e^{-\frac{1}{2 \mu \pi i} \Gamma\left(\frac{1}{2}\right)}}{\Gamma\left(\frac{3 \pm \sigma}{4}\right)}\left[1+\frac{E(k) \ln k}{k}\right] \\
\beta_{i, \nu}(k) & =\frac{\Gamma(1+2 j) e^{\frac{1}{4}((2+4 i)(\nu+1) \pm \sigma] \pi i}}{\Gamma\left(\frac{2+4 \mp \sigma}{4}\right)}\left[1+\frac{E(k) \ln k}{k}\right] \\
\beta_{i, \nu+1}(k) & =\frac{\Gamma(1+2 j) e^{\frac{1}{4}[(2+4 j) \nu \pm \sigma] \pi i}}{\Gamma\left(\frac{2+4 j \pm \sigma}{4}\right)}\left[1+\frac{E(k) \ln k}{k}\right] .
\end{aligned}
$$

To find the form of a solution $u_{\mu}(z, k), \mu=\nu$ or $\nu+1$, when $\xi(z, k) \varepsilon \Xi_{m}$, one replaces the solutions $u_{\frac{1}{2}}$ and $u_{-\frac{1}{2}}$ in the identity (7.la) by their equivalents in the identity (7.lb) with $\nu=m$.

8. Identification of $W_{k, m}(x)$ and $W_{-k, m}\left(e^{-\pi i} x\right)$. The connection between the Whittaker functions and the solutions $U_{i}(z, k)$ of (1.3) has already been found in $§ 3$. These solutions may be expressed as linear combinations of $u_{0}(z, k)$ and $u_{1}(z, k)$, namely,

$$
U_{i}(z, k)=B_{0, i}(k) u_{0}(z, k)+B_{1, i}(k) U_{1}(z, k), \quad i=1,2 .
$$

The asymptotic behavior of $U_{i}, u_{0}$, and $u_{1}$ is given by (3.2), (4.15), and (6.6)

5 In the formulas for the $\beta$ 's the upper sign is to be used if $\nu$ is even, the lower if $\nu$ is odd. 
when $z \varepsilon S \cap T$, these regions being defined by (2.5) and (2.6). On the basis of this information the constants $B_{0, i}$ and $B_{1, i}$ may be evaluated.

Let it be assumed that $z \varepsilon S \cap T$. For such $z, 0 \leqq \arg \xi(z) \leqq \pi+\epsilon$ and $|\xi(z)|>M$. It also may be seen from (2.7), (2.8), and (3.1) that

$$
e^{i \zeta}=e^{\frac{1}{2}(\sigma z-\xi)}\left(e^{z}-1\right)^{-\frac{1}{2} \sigma} \text { and } \psi(z, k)=\left(e^{z}-1\right)\left[1+\frac{E(z, k)}{k}\right] .
$$

By replacing the solutions involved in (8.1) when $i=1$ by their asymptotic forms and then making use of (8.2), the formula

$$
\begin{aligned}
& e^{i \zeta}=k^{-\frac{1}{k}(\sigma+1)} 2^{-\frac{1}{2}} B_{0,1} e^{i \zeta}\left[1+\frac{E(z, k) \ln k}{k}\right] \\
& +k^{\frac{1}{4}(\sigma-1)} 2^{-\frac{1}{2}} B_{1,1} e^{-i \zeta-\frac{1}{6} \sigma \pi i}\left[1+\frac{E(z, k) \ln k}{k}\right]
\end{aligned}
$$

can be obtained. The range of $\arg \zeta$ is from $\frac{1}{2} \pi$ to $\frac{3}{2} \pi$. If $R(i \zeta)>0$ and $e^{i \zeta}$ is large, $e^{-i \zeta}$ is small. Thus in (8.3) it must be that

$$
B_{0,1}(k)=2^{\frac{1}{k}} k^{\frac{1}{2(\sigma+1)}}\left[1+\frac{E(k) \ln k}{k}\right] .
$$

Therefore, since $B_{1,1}$ is independent of $z$,

$$
B_{1,1}(k)=e^{2 i \zeta_{p}} E(k) k^{-\frac{1}{2}(3+\sigma)} \ln k
$$

for any $\zeta_{p}=\zeta\left(z_{p}, k\right), z_{p} \varepsilon S \cap T$. Choose $z_{p}$ such that $R\left(i \zeta_{p}\right) \leqq \Re(i \zeta(z))$ for all $z \varepsilon S \cap T$. Then the minimum value of $\mathbb{R}(i \zeta(z))$ for $z \varepsilon S$ is greater than or equal to $R\left(i \zeta_{p}\right) . R\left(i \zeta_{p}\right)$ is negative; hence, the term $B_{1,1}(k) u_{1}(z, k)$ in (8.1) is asymptotically negligible for all $z \varepsilon S$. In fact, it may be shown that

$$
U_{1}(z, k)=2^{\frac{1}{2} k^{\frac{1}{2}(\sigma+1)}} u_{0}(z, k)\left[1+\frac{E(z, k) \ln k}{k}\right]+e^{i \zeta_{p}} \frac{E(z, k) \ln k}{k}
$$

for $z \varepsilon S$ and $|k|>N$. Similar reasoning leads to the conclusion that in the same circumstances

$$
U_{2}(z, k)=2^{\frac{1}{2}} e^{\frac{1}{2 \sigma \pi} i} k^{\frac{1}{(1-\sigma)}} u_{1}(z, k)\left[1+\frac{E(z, k) \ln k}{k}\right]+e^{-i \zeta_{a}} \frac{E(z, k) \ln k}{k},
$$

where $\zeta_{q}=\zeta\left(z_{q}, k\right)$ and $z_{q}$ is such that $\mathcal{R}\left(i \zeta_{q}\right) \geqq \mathcal{R}(i \zeta(z))$ for $z \varepsilon S$.

The behavior of the solutions $u_{0}(z, k)$ and $u_{1}(z, k)$ for $z \varepsilon S$ is given by the several relations (4.15), (5.4), (6.5), (6.6), (7.1), and (7.2) and the remarks made immediately after Theorem 3. The formulas (3.3), (3.7), (3.8), (8.4), and (8.5) may thus be used to establish the following theorems.

Theorem 4. When $|\xi| \leqq M,|k|>N,|\sigma|<N$, and the conditions (2.1) are fulfilled, 


$$
\begin{array}{r}
W_{k, m}(x)=C_{1}\left(\frac{x}{2 k}\right)^{\frac{1}{3}}\left\{\left[\frac{\Gamma\left(-\frac{1}{2}\right)}{\Gamma\left(\frac{1+\sigma}{4}\right)} y_{\frac{1}{2}}(z, k)+\frac{\Gamma\left(\frac{1}{2}\right)}{\Gamma\left(\frac{3+\sigma}{4}\right)} y_{-\frac{1}{2}}(z, k)\right]\left[1+\frac{E(z, k) \ln k}{k}\right]\right. \\
\left.+e^{i \zeta_{\nu}} E(z, k) k^{-\frac{1}{2}(\sigma+1)}\right\},
\end{array}
$$

and

$$
\begin{aligned}
W_{-k, m}\left(e^{-\pi i} x\right)=C_{2}\left(\frac{x}{2 k}\right)^{\frac{1}{3}}\left\{\left[\frac{-i \Gamma\left(-\frac{1}{2}\right)}{\Gamma\left(\frac{1-\sigma}{4}\right)} y_{\frac{1}{k}}(z, k)+\frac{\Gamma\left(\frac{1}{2}\right)}{\Gamma\left(\frac{3-\sigma}{4}\right)} y_{-\frac{1}{2}}(z, k)\right]\right. \\
\left.\cdot\left[1+\frac{E(z, k) \ln k}{k}\right]+e^{-i \zeta} E(z, k) k^{\frac{1}{2}(\sigma-1)}\right\},
\end{aligned}
$$

wherein $C_{1}=(2 k / e)^{k} 2^{\frac{1}{3}} k^{\frac{1}{4}(\sigma+1)}, C_{2}=(e / 2 k)^{k} 2^{\frac{1}{1}} e^{\frac{1}{4}(\sigma-1+4 k) \pi i} k^{\frac{1}{4(1-\sigma)}}$,

$$
\begin{aligned}
\left.y_{1}(z, k)=\Psi(z) e^{-\frac{1}{2} \xi^{\frac{1}{2}}\left\{{ } _ { 1 } F _ { 1 } \left(\frac{3+\sigma}{4}\right.\right.} ; \frac{3}{2} ; \xi\right)\left[e^{-\theta}+\frac{\sinh \theta}{\xi}\right] \\
\left.+\frac{3+\sigma}{3} \sinh \theta_{1} F_{1}\left(\frac{7+\sigma}{4} ; \frac{5}{2} ; \xi\right)\right\}\left\{1+\frac{E(z, k)}{k}\right\},
\end{aligned}
$$

and

$$
\begin{aligned}
y_{-\frac{1}{2}}(z, k)=\Psi(z) e^{-\frac{1}{2} \xi}\left\{e^{-\theta}{ }_{1} F_{1}\left(\frac{\sigma+1}{4} ; \frac{1}{2} ; \xi\right)\right. & \\
& \left.+(\sigma+1) \sinh \theta_{1} F_{1}\left(\frac{\sigma+5}{4} ; \frac{3}{2} ; \xi\right)\right\}\left\{1+\frac{E(z, k)}{k}\right\} .
\end{aligned}
$$

The quantities $z, \sigma, \xi, \Psi$, and $\theta$ are defined by (1.2), (1.4), (2.7), (4.3c), and (4.9a), respectively. The definitions of $\zeta_{p}$ and $\zeta_{\alpha}$ are given in the second paragraph of this section.

Theorem 5. When $|\xi| \geqq M,|k|>N,|\sigma|<N$, the conditions (2.1) are fulfilled, and $\xi \varepsilon \Xi_{\nu}, W_{k, m}(x)=F_{\nu}(x, k)+e^{-\frac{1}{2} x_{p}} x_{\nu}^{k} E(x, k)$ and $W_{-k, m}\left(e^{-\pi i} x\right)=G_{\nu}(x, k)$ $+e^{\frac{1}{2} x} x x_{a}^{-k} E(x, k)$, where $\nu=0, \pm 1, \pm 2$, and

$$
\begin{aligned}
F_{2}(x, k) & =c_{1} e^{-\frac{1}{2} \sigma \pi i} f_{1}(x)-e^{\sigma \pi i} f_{2}(x), & G_{2}(x, k) & =f_{1}(x)+c_{2} e^{\sigma \pi i} f_{2}(x), \\
F_{1}(x, k) & =c_{1} e^{-\frac{1}{2} \sigma \pi i} f_{1}(x)+f_{2}(x), & G_{1}(x, k) & =f_{1}(x), \\
F_{0}(x, k) & =f_{2}(x), & G_{0}(x, k) & =f_{1}(x), \\
F_{-1}(x, k) & =f_{2}(x), & G_{-1}(x, k) & =f_{1}(x)-c_{2} f_{2}(x), \\
F_{-2}(x, k) & =-c_{1} e^{\frac{1}{\sigma \sigma \pi} i} f_{1}(x)+f_{2}(x), & G_{-2}(x, k) & =-e^{\sigma \pi i} f_{1}(x)-c_{2} f_{2}(x) .
\end{aligned}
$$


In these formulas $x_{p}\left[x_{q}\right]$ is such that $\zeta\left(z\left(x_{p}\right), k\right)=\zeta_{p}\left[\zeta\left(z\left(x_{q}\right), k\right)=\zeta_{q}\right]$; also

and

$$
\begin{gathered}
c_{1}=\frac{2 \pi i(2 k)^{2 k} k^{\frac{1}{2} \sigma} e^{-(\pi i+2) k}}{\Gamma\left(\frac{1+\sigma}{4}\right) \Gamma\left(\frac{3+\sigma}{4}\right)}, \quad c_{2}=\frac{2 \pi i(2 k)^{-2 k} k^{-\frac{1}{2} \sigma} e^{(\pi i+2) k}}{\Gamma\left(\frac{1-\sigma}{4}\right) \Gamma\left(\frac{3-\sigma}{4}\right)}, \\
f_{1}(x)=x^{-k}\left(1-\frac{2 k}{x}\right)^{\frac{1}{2}(\sigma-1)} e^{k \pi i+\frac{1}{2} x}\left[1+\frac{E(x, k) \ln k}{k}\right],
\end{gathered}
$$

$$
f_{2}(x)=x^{k}\left(1-\frac{2 k}{x}\right)^{-\frac{1}{2}(\sigma+1)} e^{-\frac{1}{2} x}\left[1+\frac{E(x, k) \ln k}{k}\right] \text {. }
$$

It should be noted that the error terms $e^{-\frac{1}{2} x_{p}} x_{p}^{k}$ and $e^{\frac{1}{2} x_{a}} x_{\alpha}^{-k}$ are small as $|\mathcal{R}(x)|$ $\gg 2|k|$, if $x=x_{p}$ or $x_{a}, \Re\left(x_{p}\right)>0$, and $R\left(x_{a}\right)<0$. The regions $\Xi_{\nu}$ are defined by (4.8), wherein $S_{\xi}$ is the image of the region (2.5) under the mapping (2.7).

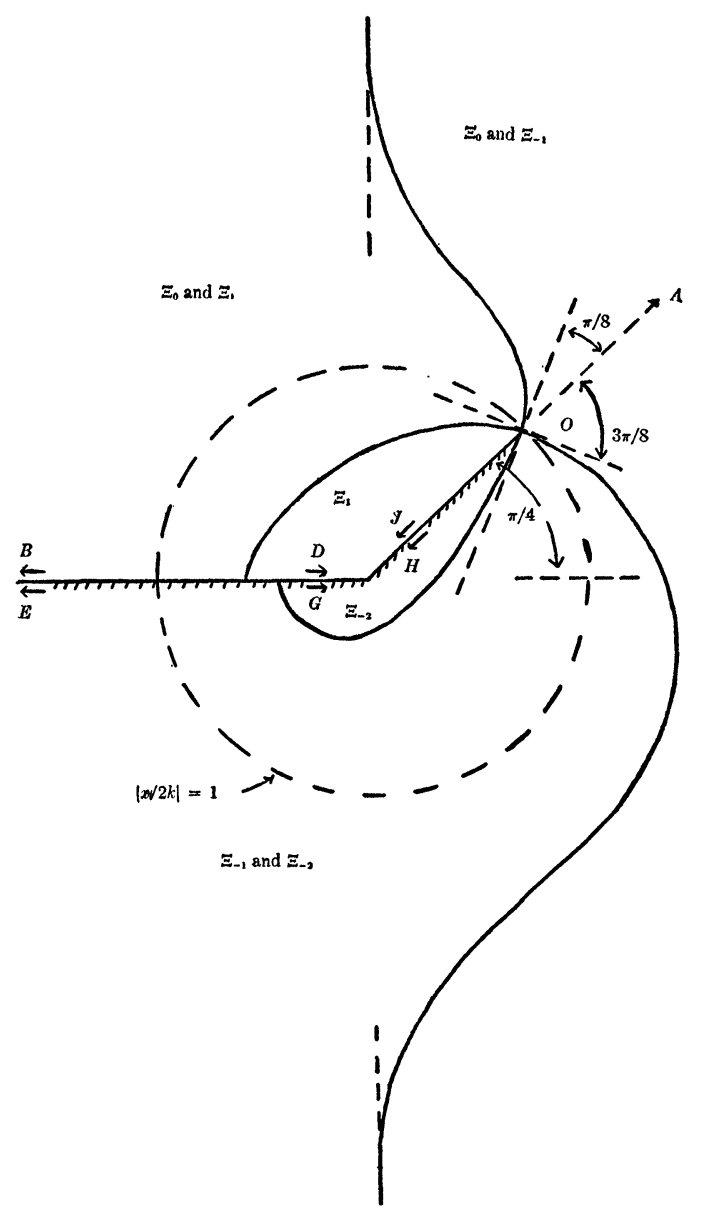

Fig. 3. The cut $x$-plane. 
Equation (3.10) may again be used to obtain asymptotic forms for the Whittaker functions $M_{k, \pm m}(x)$.

The general features of the boundaries of the images of the regions $\Xi_{\nu}$ on the $x$-plane are illustrated in Fig. 3 for $\arg k=\frac{1}{4} \pi$. Near the point $x=2 k, \arg (x-2 k)$ $\approx \frac{1}{2}(\arg \xi+\arg k) ;$ when $|x / 2 k|$ is large, $\arg x \approx \arg \xi$. The notation of Fig. 3 conforms to that of Fig. 2 . The boundaries of the images of the regions $\Xi_{\nu}$ are indicated by solid lines. The presence of a symbol $\Xi_{\nu}$ in a region bounded by solid lines means the region lies in the image of $\Xi_{\nu}$. The boundaries $|x / 2 k|=e^{\star \alpha}$ are not indicated in the figure.

9. Equivalent results in Kummer's notation and for Laguerre polynomials. As stated in the introduction a study of the behavior of the Whittaker functions $W_{k, m}(x)$ and $W_{-k, m}\left(e^{-\pi i} x\right)$ as $|k|,|m| \rightarrow \infty$, while $\left(m^{2}-k^{2}\right) / k$ is bounded is equivalent to the study of the behavior of the Kummer functions ${ }_{1} F_{1}(a ; c ; x)$ and ${ }_{1} F_{1}(1+a-c ; 2-c ; x)$ as $|c| \rightarrow \infty$. In this instance $\sigma=\left[2 a(c-a)-c+\frac{1}{2}\right]$ $/(c-2 a)$. If $a$ is fixed and $|c| \rightarrow \infty$ or if both $|c|$ and $|a| \rightarrow \infty$ while $c-a$ is bounded, then $\sigma$ remains bounded. For the Laguerre polynomials $L_{n}^{\alpha}(x) \sigma=$ $-\left[n^{2}+(\alpha+1) n+\frac{1}{4}(2 \alpha+1)\right] /\left[n+\frac{1}{2}(\alpha+1)\right]$. When $n$ is bounded and $|\alpha| \rightarrow \infty$, $\sigma$ remains bounded. In each of these cases, therefore, Theorems $1,2,4$, and 5 apply. Thus from Theorem 1, for example,

$$
L_{n}^{\alpha}(x)=\frac{(-1)^{n}}{n !}\left(1-\frac{2 n+\alpha+1}{x}\right)^{-\frac{1}{2}(\sigma+1)} x^{n+\frac{1}{2}(\alpha+1)}\left[1+\frac{E(x, \alpha)}{\alpha}\right]
$$

when $\mid \arg \left(n+\frac{1}{2}(\alpha+1) \mid \leqq \pi, 0 \leqq \arg x \leqq \pi\right.$, and $|x /(2 n+\alpha+1)|>1+\epsilon$, $\epsilon>0$.

\section{References}

1. Bateman Manuscript Proj., Higher transcendental functions, Vol. 1, McGraw-Hill, 1953.

2. H. Buchноцz, Die konfluente hypergeometrische Funktion, Springer-Verlag, Berlin, 1953.

3. C. Chang, B. Chu, \& V. O'Brien, An asymptotic expansion of the Whittaker function $W_{k, m}(z)$, J. of Rat. Mech. and Anal. 2 (1949) pp. 125-135.

4. - Asymptotic expansion of the Whittaker's function $W_{k, m}(z)$ for large values of $k, m, z$, J. of the Franklin Inst. 255 (1953) pp. 215-236, 319-331.

5. A. ERDELlyI, Asymptotische Darstellung der Whittakerschen Funktionen für grosse reelle Werte des Argumentes und der Parameter, Časopis pro Péstování. Mat. a Fys. 67 (1938) pp. 240-248.

6. N. KAZARINOFF, Asymptotic expansions for the Whittaker functions of large complex order $m$, Trans. Amer. Soc. 78 (1955) pp. 305-328.

7. H. Kober, Dictionary of conformal representations, Dover, New York, 1952.

8. R. LANGER, The asymptotic solutions of ordinary linear differential equations of the second order with special reference to a turning point, Trans. Amer. Math. Soc. 67 (1949) pp. 461-490.

9. R. McKelver, The solutions of second order ordinary differential equations about a turning point of order two, Trans. Amer. Soc. 79 (1955) pp. 103-123.

10. F. Tricomi, Funzioni ipergeometriche confluenti, Edizioni Cremonese, Roma, 1954.

Purdue University

Lafayette, Indiana 